\title{
Long non-coding RNA LNC_000641 regulates pseudorabies virus replication
}

\author{
Linlin Fang ${ }^{1}$, Yanni Gao ${ }^{1,2}$, Xing Liu ${ }^{1,2}$, Juan Bai ${ }^{1,2}$, Ping Jiang ${ }^{1,2}$ and XianWei Wang ${ }^{1,2^{*}}$ (1)
}

\begin{abstract}
Long non-coding RNAs (IncRNAs) are a new arm of gene regulatory mechanism as discovered by sequencing techniques and follow-up functional studies. The IncRNAs regulation of pseudorabies virus (PRV) infection has rarely been reported so far. Using RNA sequencing analysis, 225 IncRNAs with significant altered expressions in 3D4/21 cells infected with PRV (ZJ01) were identified. Five IncRNAs upregulated in PRV-infected cells were verified in cells infected with different PRV strains by qRT-PCR. By down- and up-regulation of Inc641, the accelerating effect of Inc641 on PRV replication was confirmed. Furthermore, we found that Inc641 regulated PRV replication by inhibiting the JAK-STAT1 pathway. This study suggests that Inc641 could be a new host factor target for developing antiviral therapies against PRV infection.
\end{abstract}

Keywords: Pseudorabies virus, Long non-coding RNAs (IncRNAs), Anti-viral activity, IFN-alpha, JAK/STAT1

\section{Introduction}

Pseudorabies virus (PRV), known as Aujeszky's disease virus or suid herpesvirus 1 , is a member of the alphaherpesvirinae subfamily and threatens pig production [1]. PRV is a highly infectious and lethal pathogen in pigs responsible for Aujeszky's disease, which causes abortions and stillbirths in sows, central nervous system disorders in young piglets, and respiratory disease in older pigs. PRV genome is a double-stranded DNA with a length of $142334 \mathrm{bp}[2,3]$. The mature virion, or infectious viral particle, consists of four morphologically distinct structural components: the central core contains the linear double-stranded DNA genome of the virus; the DNA is enclosed within a protective icosahedral capsid to form a nucleocapsid; the capsid is embedded in a protein matrix known as the tegument; finally, the tegument is surrounded by the envelope, a lipid membrane

\footnotetext{
*Correspondence: xwwang@njau.edu.cn

${ }^{1}$ Key Laboratory of Animal Diseases Diagnostic and Immunology, Ministry of Agriculture, MOE Joint International Research Laboratory of Animal Health and Food Safety, College of Veterinary Medicine, Nanjing Agricultural University, Nanjing 210095, China

Full list of author information is available at the end of the article
}

containing several viral glycoproteins $[4,5]$. PRV infection impairs interferon (IFN) signaling to establish persistent infection in host cells, by suppressing IFN-induced upregulation of STAT1 phosphorylation and various interferon-stimulated genes (ISGs) [6].

Host genome DNA sequencing is of importance for understanding host evolution, disease origin, and the interplay between environment and heredity. The emergence of high-throughput sequencing technologies has had the greatest impact on the expanding world of noncoding RNAs. The first transcriptome analyses led to the unexpected discovery that while most of the genome is transcribed, only $2 \%$ of the genome is transcribed into mRNAs encoding proteins. It was apparent that the majority of the genome was transcribed into noncoding RNAs [7]. Among the noncoding genome, long noncoding RNAs (lncRNAs) constitutes a particularly rich category. lncRNA is a class of transcripts with more than $200 \mathrm{bp}$ in length without encoding possibility in eukaryotes $[8,9]$. Some estimates suggest that the human genome contains more than 90000 genes and approximately 60000 of them are lncRNAs, while other estimates suggest that the number of lncRNA genes could reach closer to 200000 [9].

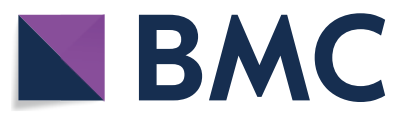

(C) The Author(s) 2021. This article is licensed under a Creative Commons Attribution 4.0 International License, which permits use, sharing, adaptation, distribution and reproduction in any medium or format, as long as you give appropriate credit to the original author(s) and the source, provide a link to the Creative Commons licence, and indicate if changes were made. The images or other third party material in this article are included in the article's Creative Commons licence, unless indicated otherwise in a credit line to the material. If material is not included in the article's Creative Commons licence and your intended use is not permitted by statutory regulation or exceeds the permitted use, you will need to obtain permission directly from the copyright holder. To view a copy of this licence, visit http://creativeco mmons.org/licenses/by/4.0/. The Creative Commons Public Domain Dedication waiver (http://creativecommons.org/publicdomain/ zero/1.0/) applies to the data made available in this article, unless otherwise stated in a credit line to the data. 
LncRNAs play important roles in many biological processes. The roles of lncRNAs in viral infections have been documented $[10,11]$. Some differentially expressed lncRNAs regulate inflammatory innate responses and pathogen evasion or survival during host-pathogen interactions [12-14]. Negative regulator of antiviral response (NRAV) is a lncRNA that is downregulated by various viruses including influenza virus, sendai virus, muscovy duck reovirus, and herpes simplex virus [15]. Overexpression of NRAV increases virus replication whereas knockdown of NRAV has an opposite effect.

The innate immune response is a host first line of defense against invading viruses [16]. Type I interferons (IFNs), primarily IFN- $\alpha / \beta$, are produced by host cells as "early" antiviral agents $[17,18]$ and are recognized as a critical part of the host innate immune response to virus infection. Type I IFNs bind to their receptors to activate molecules downstream Janus kinase-signal transducer and activator of transcription (JAK-STAT) signaling that consequently initiates the transcription of ISGs, including noncoding transcripts, which exert a broad spectrum of antiviral effects [6]. In general, the binding of IFN $-\alpha / \beta$ to their receptors results in the cross-phosphorylation of Janus kinases (Jaks) at tyrosines, which provides docking sites for signal transducers and activators of transcription (Stats) leading to stat phosphorylation. The phosphorylated stats (pStats) then dissociate from the receptor, dimerize and translocate into the nucleus to regulate downstream gene expression [19]. LncRNAs such as THRIL [12] lincRNA-Cox2 and Lethe [20] have been shown to regulate gene expression in innate immune cells. LncRNAs are emerging as critical regulators of both innate and adaptive immunity [21]. These studies suggest that lncRNAs play a crucial role in virus pathogenesis. However, the regulation by lncRNAs of PRV infection is still not well known.

In the present study, using RNA sequencing analysis, we showed that the expression of 225 lncRNAs was significantly altered in 3D4/21cells infected with PRV. According to an analysis of differential expression between mock-infected and PRV-infected cells, 126 host lncRNAs were significantly upregulated and 99 host lncRNAs were significantly downregulated in the latter group.

\section{Materials and methods}

\section{Cells, viruses and reagents}

Porcine alveolar macrophages cells (3D4/21, ATCC ${ }^{\circledR}$ CRL-2843 ${ }^{\mathrm{TM}}$ ), porcine kidney cells (PK-15, ATCC ${ }^{\circledR}$ CCL$33^{\mathrm{TM}}$ ), porcine testis cells (ST, ATCC ${ }^{\circledR}$ CRL-1746 ${ }^{\mathrm{TM}}$ ) were stored in the laboratory. 3D4/21 cells were cultured in RPMI-1640 medium (Gibco, USA) supplemented with $10 \%$ fetal bovine serum (FBS; LONSERA) and $0.1 \mathrm{mM}$ non-essential amino acid (NAA), PK-15 cells and ST cells were cultured in Dulbecco's modified Eagle's medium (Corning, USA) supplemented with $10 \%$ fetal bovine serum (FBS; LONSERA) at $37^{\circ} \mathrm{C}$ in a humidified atmosphere containing $5 \% \mathrm{CO}_{2}$. Two PRV strains (ZJ01 and LA) were used. PRV was proliferated in PK-15 cells and was stored at $-80^{\circ} \mathrm{C}$. The highly pathogenic PRV strain ZJ01, which is maintained in our laboratory, was used for all experiments. The PRV strain LA was also used, as specifically mentioned by name (LA, a classical strain).

Anti-PRV gB-protein monoclonal antibody (1B1, prepared and stored in our laboratory), anti-GAPDH antibody (Proteintech, USA), anti-P-STAT1 antibody (Cell Signal Technology, USA), anti-STAT1 antibody (Cell Signal Technology, USA), anti-p-JAK1 antibody (Affinity, USA) and anti-JAK1 antibody (Affinity, USA) were used in the study.

\section{RNA sequencing and data analysis}

3D4/21 cells were infected with ZJ01 PRV strain at a $0.5 \mathrm{MOI}$; uninfected 3D4/21 cells were used as control. $22 \mathrm{~h}$ post-infection, cells were harvested by scraping and then put into Trizol (Invitrogen, USA). Three parallel replicates were performed for uninfected and infected cells. RNA isolation and sequencing were performed by Novogene Bioinformatics Technology Co., Ltd. (Beijing, China). Transcripts with a $P$-adjust $<0.05$ were assigned as differentially expressed. GO and KEGG analyses were performed to understand the effect of PRV infection on cell biological processes, molecular function, and cellular components.

\section{RNA extraction and quantitative qRT-PCR}

Total RNA was extracted from cells using a Total RNA Kit I (Omega Bioek). RNA purity was then detected by NanoDrop 2000 (Thermo Scientific, USA). The reverse transcription was performed using a HiScript II 1st Strand cDNA Synthesis Kit (Vazyme, China) following the manufacturer's instructions. Quantitative RT-PCR was performed using AceQ ${ }^{\circledR}$ qPCR SYBR ${ }^{\circledR}$ Green Master Mix (Vazyme, China) according to the manufacturer's instructions. The quantity of cDNA was $100 \mathrm{ng}$ and the final concentration of primers was $0.2 \mu \mathrm{M}$. The qRT-PCR thermal conditions were $95^{\circ} \mathrm{C}$ for $5 \mathrm{~min}$, followed by 40 cycles of $95^{\circ} \mathrm{C}$ for $10 \mathrm{~s}$ and $60{ }^{\circ} \mathrm{C}$ for $30 \mathrm{~s}$, then $95^{\circ} \mathrm{C}$ for $30 \mathrm{~s}, 60^{\circ} \mathrm{C}$ for $60 \mathrm{~s}, 95^{\circ} \mathrm{C}$ for $15 \mathrm{~s}$. Data are presented as the fold change in gene expression normalized to $\beta$-actin and relative to the mock-infected control. Each reaction was performed in triplicate, and the data are calculated as the mean $(M) \pm S E M$. The sequences of primer for genes are shown in Table 1. 
Table 1 Primer sequences used for qRT-PCR analysis

\begin{tabular}{lll}
\hline Primer & Sequence $\left(5^{\prime} \rightarrow 3^{\prime}\right.$ ) & Product \\
\hline IFN-alpha-Fwd & TCCAGAAACCTGCAAGACAG & IFN-alpha \\
IFN-alpha-Rev & ATGGGCTTGTTAGTCTGTGAG & \\
IFN-beta-Fwd & ACCACAGCTCTTCCATGAG & IFN-beta \\
IFN-beta-Rev & CAGGGACCTCAAAGTTCATCC & \\
IFN-gamma-Fwd & AATGGTAGCTCTGGGAAACTG & IFN-gamma \\
IFN-gamma-Rev & ACTTCTCTTCCGCTTTCTTAGG & \\
18s rRNA-Fwd & CGTTGATTAAGTCCCTGCCCTT & 18s rRNA \\
18s rRNA-Rev & TCAAGTTCGACCGTCTTCTCAG & \\
U2snRNA-Fwd & CATCGCTTCTCGGCCTTTTG & U2snRNA \\
U2snRNA-Rev & TGGAGGTACTGCAATACCAGG & \\
gB-Fwd & GTCCGTGAAGCGGTTCGTGAT & PRV-gB \\
gB-Rev & ACAAGTTCAAGGCCCACATCTAC & \\
B-actin-Fwd & GTGATCTCCTTCTGCATCCTGTC & B-actin \\
B-actin-Rev & CTCCATCATGAAGTGCGACGT & \\
Inc641-Fwd & CAGGCATAGAGGGTTAAGGAC & Inc641 \\
Inc641-Rev & ACGCTTTGCATGTGGAATTC & \\
ALD1114-Fwd & GGTGGGCAAAAGAACTTAGTG & ALD1114 \\
ALD1114-Rev & GATAAGAACACGGCTCCCTG & \\
Inc1007-Fwd & CTCAGTGGGTAATGATCCGG & Inc1007 \\
Inc1007-Rev & CATATGGAGGTTCCCAGGTTAG & \\
ALD8954-Fwd & AAGTGGTACAAGACAGTGTGG & ALD8954 \\
ALD8954-Rev & GGAGGTTGGAGGTAAAAGAC & \\
Inc1059-Fwd & TCTTGGGCTCTGCAAATGAG & Inc1059 \\
Inc1059-Rev & AAGGCTCCTTCTGTCTTGTTC & \\
\hline
\end{tabular}

\section{Western blot analysis}

Cells were lysed on ice for $10 \mathrm{~min}$ in lysis buffer (Beyotime, China), then resolved by $10 \%$ SDS-PAGE and transferred onto nitrocellulose membrane. The membrane was blocked with $10 \%$ low-fat milk for $2 \mathrm{~h}$ at room temperature and then incubated with antibodies: anti-PRV gB-protein (1B1, 1:5000), anti-GAPDH (1:5000), antiP-STAT1 (1:5000), anti-STAT1 (1:1000), anti-p-JAK1 (1:1000), anti-JAK1 (1:1000) for $2 \mathrm{~h}$ at room temperature. Membranes were incubated with HRP-conjugated goat anti-mouse and anti-rabbit IgG $(\mathrm{H}-\mathrm{L})$ secondary antibodies (1:1000; Beyotime, China). The proteins on the membranes were observed using the Chemistar Highsig ECL Western blotting substrate (Tanon, China) and developed on a Tanon 5200 system (Tanon, China).

\section{Indirect immunofluorescence assay (IFA)}

PRV-infected cells were fixed with $4 \%$ paraformaldehyde for $15 \mathrm{~min}$ and then permeabilized with $0.1 \%$ Triton X-100. After three washes with PBS, cells were incubated with an anti-PRV gB-protein monoclonal antibody (1B1, 1:2500, made in our laboratory) for $1 \mathrm{~h}$ at $37{ }^{\circ} \mathrm{C}$. Cells were washed three times with PBS and then incubated with Alexa Fluor 488-conjugated goat anti-mouse IgG (H-L) $\left(1: 200\right.$, Proteintech) for $1 \mathrm{~h}$ at $37^{\circ} \mathrm{C}$ in the dark. Nuclei were stained with 4',6-diamidino-2-phenylindole (DAPI; $100 \mathrm{ng} / \mathrm{mL}$, Beyotime, Nanjing, China) for $5 \mathrm{~min}$ at room temperature. Immunofluorescence was observed using an inverted fluorescence microscope (Zeiss Axio Observer; Zeiss, Germany).

\section{Plasmid construction}

Total RNA was extracted from 3D4/21 cells using a Total RNA Kit I (Omega Biotek, Norcross, GA, USA), and cDNA synthesis was performed with SuperScript III Reverse Transcriptase (Invitrogen). Lnc641 was generated by PCR amplification of cDNA from 3D4/21 cells with the oligonucleotide pair KpnI lnc641 5'-CGC GGT ACC ATG CAA GGA CTG AGG GAG AGA GAG CGC CGA-3' and XhoI lnc641 5'-GCG CTC GAG CTA TGC ATG GCC ATG CAA GGA AAT CGG TGT T-3' The sequence of the amplification product was compared to that in the transcriptome results for verification, restriction digested, and cloned into the pcDNA3.1 $(+)$ vector to produce pcDNA3.1-lnc641.

\section{Plasmid transfection and virus challenge}

To determine the effects of lncRNA on PRV replication, 3D4/21 cells plated in 24-well plates were transfected with $0,0.4,0.8,1 \mu \mathrm{g}$ of pcDNA3.1-lnc641 using Lipofectamine 3000 (Invitrogen) according to the manufacturer's recommendations. Twenty-four hours after transfection, the cells were infected with PRV (0.01 MOI) and then harvested for qRT-PCR, Western blotting and IFA at $24 \mathrm{hpi}$.

\section{Small interfering RNA assays}

Three siRNAs targeting lnc641 were designed and synthesized by Invitrogen. The primer sequences used were as follows: siRNA1 (5'-GAC GAA CUU GAC AAG ACU AdTdT-3', 5'-UAG UCU UGU CAA GUU CGU CdTdT-3'); siRNA2 (5'- GGA AGG CUA AGA AGG AGA AdTdT-3' ${ }^{\prime}$,'-UUC UCC UUC UUA GCC UUC CdTdT-3'). 3D4/21 cells plated in 24-well plates were transfected with siRNAs or negative control (NC) using Lipofectamine 3000 Transfection Reagent (Invitrogen), following the manufacturer's instructions. After $36 \mathrm{~h}$, the cells were infected with PRV (0.01 MOI) for $24 \mathrm{~h}$. Cells were harvested for qRT-PCR, TCID 50 , Western blot and IFA.

\section{Virus titration}

3D4/21 cells grown in 96-well plates were infected with tenfold serial dilutions of PRV samples in four replicates. After $1 \mathrm{~h}$ at $37^{\circ} \mathrm{C}$, the culture medium was replaced with 
fresh DMEM. The plates were incubated for $72 \mathrm{~h}$ at $37^{\circ} \mathrm{C}$. The PRV titers were calculated using the Reed-Muench method.

\section{Isolation of cytoplasmic and nuclear RNAs}

Cytoplasmic and nuclear fractions were prepared from 3D4/21 cells using PARIS ${ }^{\mathrm{TM}}$ Kit (Invitrogen, USA). cDNA was prepared using $1 \mu \mathrm{g}$ RNA and qRT-PCR was performed to analyze both cellular fractions using primers of $\beta$-actin, lnc641, U2snRNA and 18sRNA. The expression of mRNA or lncRNA in nucleus and cytoplasm was calculated with the equation $2^{-\Delta c t}$. The percentage of each RNA in the nucleus and cytoplasm was calculated.

\section{Statistical analyses}

GraphPad Prism 7.0 software (GraphPad, La Jolla, CA, USA) was used to analyze all statistical data via one-way analysis of variance. Differences between two groups were considered statistically significant when the $P$-value was $<0.05$, highly significant at $P<0.01$, and extremely significant at $P<0.0001$.

\section{Results \\ LncRNAs are differentially expressed in PRV-infected 3D4/21 cells}

To identify lncRNAs that are dysregulated during Pseudorabies virus infection, 3D4/21 cells infected with ZJ01 PRV strain at a MOI of 0.5 for $22 \mathrm{~h}$ were submitted to RNA-seq analysis. Tophat was used for read mapping and Cufflinks/Cuffdiff was used for gene expression quantification. Figure $1 \mathrm{~A}$ depicts the strategy of the experiment. Using a $P$ value of $<0.05,225$ significantly differentially expressed lncRNAs were identified in PRV-infected 3D4/21 cells (Figure 1B). Of them, 126 1ncRNAs were upregulated and 99 1ncRNAs were downregulated (Figure 1C). There were 29 upregulated and 2 downregulated lncRNAs based on a fold change of 2 or more.

Five lncRNAs were chosen for further study as they were (a) a fold change of $>2$ for upregulated lncRNAs, (b) significant changes in the expression of neighboring genes (up or down) within $10000 \mathrm{~kb}$ of lncRNAs [22] (Table 2).

The qRT-PCR was then utilized to validate the results from RNA-seq analysis. The qRT-PCR confirmed that the production of all these 5 lncRNAs were promoted by ZJ01 (Figure 2A). To further confirm the results of the RNA-seq analysis, a qRT-PCR assay was conducted to measure the expression of IncRNA in PRV-treated cells at $6 \mathrm{~h}, 12 \mathrm{~h}, 24 \mathrm{~h}$. The results showed that the expression levels of 5 groups of lncRNA increased along with PRV infection (Figure 2B). Taking together, these data indicated that PRV infection of 3D4/21 cells promoted the above 5 lncRNA production, consistent with the RNA sequencing results.

\section{Effects of different PRV strains and cell lines on IncRNA expression}

In RNA sequencing analysis, a single MOI of ZJ01 PRV strain was used to infect 3D4/21 cells. To study the effect of doses and strains of PRV on the upregulated IncRNAs, 3D4/21 cells infected with different doses of PRV ZJ01 and LA strains were used to detect the IncRNAs productions. The result showed that the lncRNAs could be produced by both ZJ01 and LA strains in a dose-dependent manner (Figure 3). However, the magnitude of induction varied among strains and lncRNAs. ALD11114 expression reached maximum at a MOI of 0.1 and $\operatorname{lnc} 1007$ expression reached maximum at a MOI of 0.5 while ALD8954, lnc641, and lnc1059 had a highest expression at a MOI of 1 . LA strain induced higher levels of 5 lncRNAs than ZJ01 strain.

To determine whether IncRNA was also produced in other cells, Porcine kidney (PK-15) cells, Porcine testis (ST) cells infected with 0.5 MOI of ZJ01 or LA for $24 \mathrm{~h}$. The results showed that the induction was more potent in ST cells than in PK-15 cells (Figure 4).

\section{Knockdown of Inc 641 by siRNAs inhibits PRV replication}

The lnc641 with the highest fold changes were selected for further characterization and functional studies. To determine the functional role of lnc641 in PRV replication, 3D4/21 cells were transfected with two siRNAs designed to be targeting lnc641 gene. After incubation for $36 \mathrm{~h}$, lnc641 RNA levels were detected by qRTPCR (Figure 5A). The results showed that siRNA1 and siRNA2 significantly downregulated lnc641 RNA levels in 3D4/21 cells. Then 3D4/21 cells were transfected with the siRNA, and then infected with ZJ01 strain at $0.01 \mathrm{MOI}$ at $24 \mathrm{~h}$ post-transfection. At $24 \mathrm{hpi}$, cells were harvested for $\mathrm{TCID}_{50}$, qRT-PCR, Western blot assay and IFA. As shown in Figures 5B-E, the replication of PRV and the expression of $g B$ were reduced to a certain extent when siRNA knocked down lnc641, compared with the cells transfected with negative control siRNA (siNC). TCID $_{50}$ assays also showed that the knockdown of lnc641 significantly decreased viral titers.

\section{Overexpression of Inc641 enhances PRV replication}

To confirm the effect of lnc641 on PRV replication, 3D4/21 cells were transfected with different doses of pcDNA3.1-lnc641 or pcDNA3.1(+) and then infected with ZJ01 (0.01 MOI). At $24 \mathrm{hpi}$, cells were harvested 
A

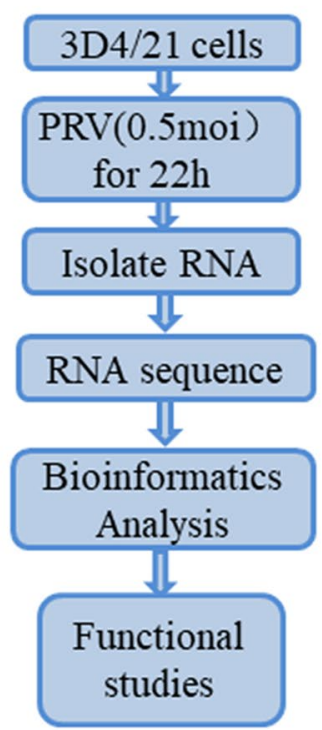

B

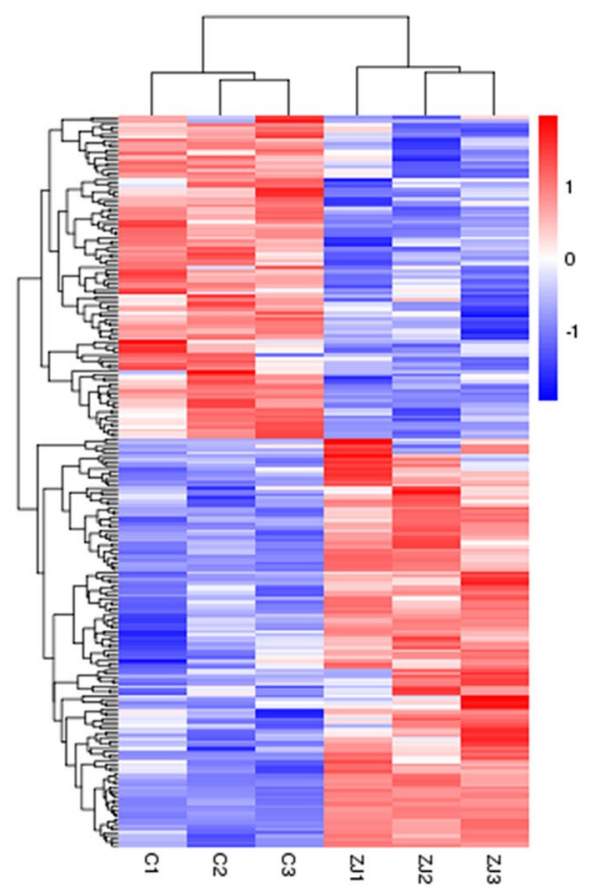

C

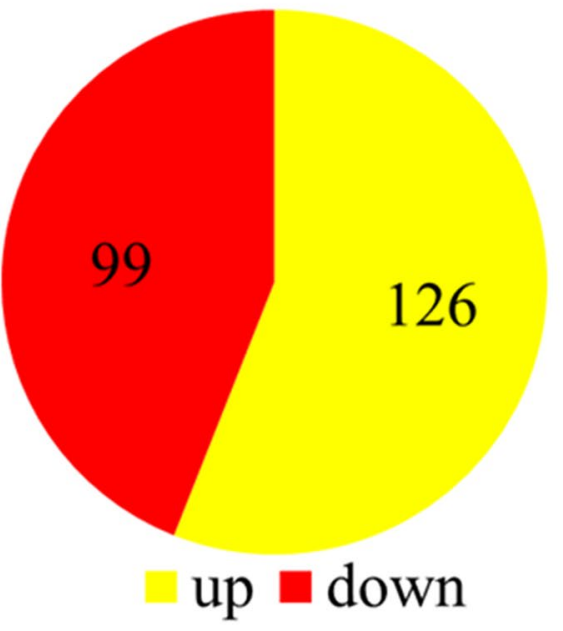

$\operatorname{lncRNA}$

Figure 1 LncRNAs are differentially regulated during PRV infection. A Flowchart of RNA-seq experiment design. B Hierarchical clustering analysis of DEGs. DEG expression levels are represented as FPKM-normalized $\log _{2}$-transformed counts. Blue indicates low relative expression, and red indicates high relative expression. C Pie charts of significantly changed IncRNAs with a $P$-value of $\leq 0.05$. Red and yellow colors denote downregulated and upregulated genes, respectively.

Table 2 Selected IncRNAs and their properties

\begin{tabular}{|c|c|c|c|c|c|}
\hline IncRNA_ID & IncRNA_Gene_ID & Gene_Type & Status & Fold change & Length (bp) \\
\hline LNC_000641 (Inc641) & XLOC_037995 & lincRNA & Novel_IncRNA & $\inf$ & 385 \\
\hline LNC_001007 (Inc1007) & XLOC_063386 & lincRNA & Novel_IncRNA & $\inf$ & 685 \\
\hline LNC_001059 (Inc1059) & XLOC_067112 & antisense_IncRNA & Novel_IncRNA & $\inf$ & 1559 \\
\hline ALDBSSCT0000011114 (ALD11114) & ALDBSSCG0000006748 & lincRNA & Annotated_IncRNA & $\inf$ & 316 \\
\hline ALDBSSCT0000008954 (ALD8954) & ALDBSSCG0000005482 & lincRNA & Annotated_IncRNA & 3.8547 & 4599 \\
\hline
\end{tabular}



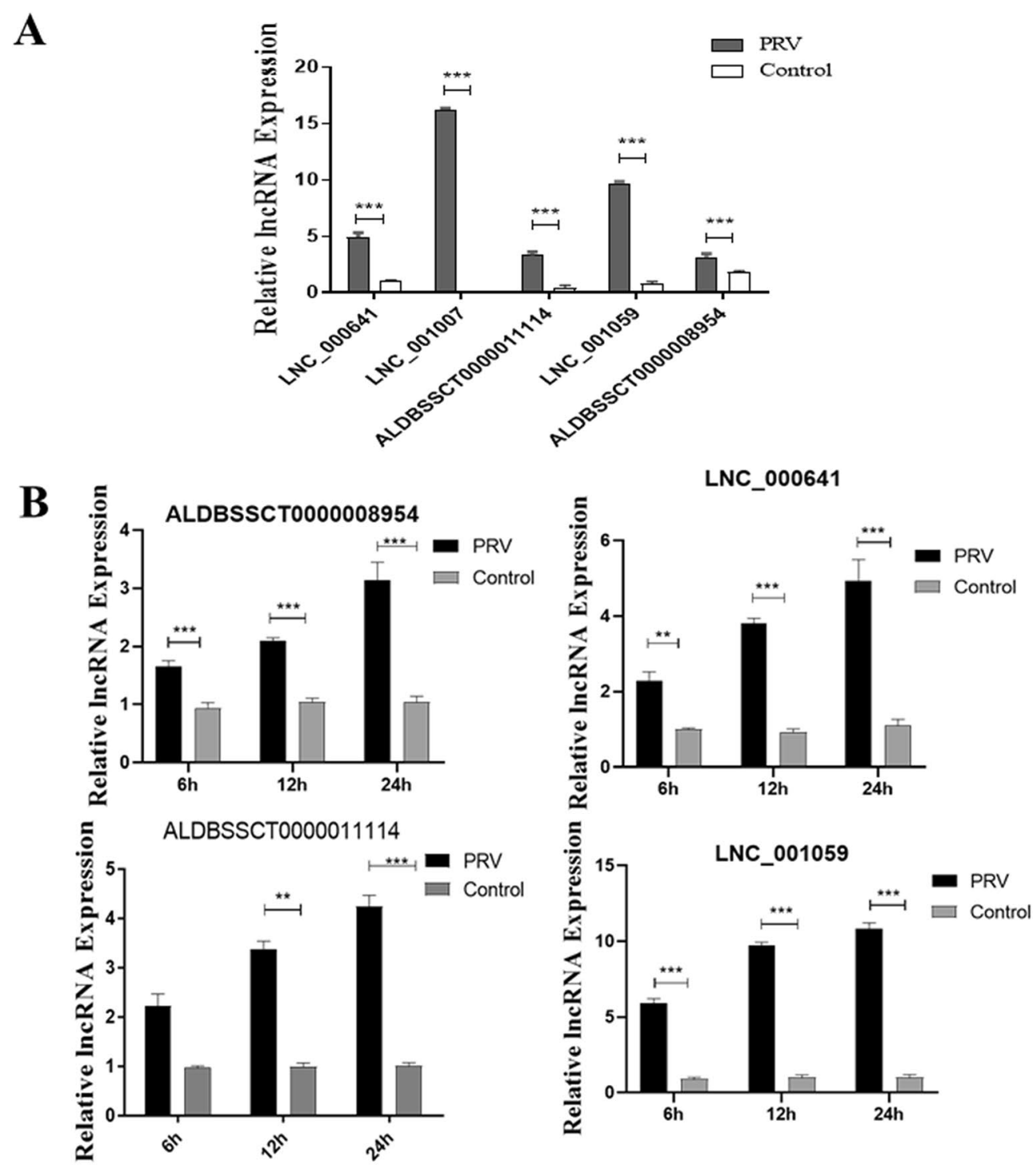

LNC_001007

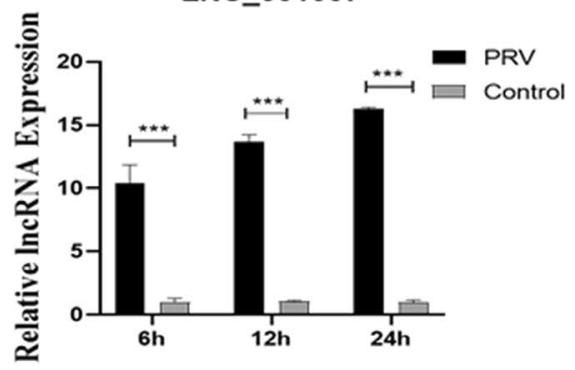

Figure 2 Validation of RNA-seq results with qRT-PCR. A 3D4/21 cells were infected with ZJ01 PRV strain at a MOl of 0.5 for $22 \mathrm{~h}$. Relative expression levels of selected IncRNAs were determined by qRT-PCR and normalized to $\beta$-actin. B qRT-PCR quantification of the expression levels of IncRNA at three time points $(6 \mathrm{~h}, 12 \mathrm{~h}, 24 \mathrm{~h})$ after PRV infection. The qRT-PCR was repeated at least three times, with each experiment performed in triplicate. ${ }^{* * *} P<0.001 ;{ }^{* *} P<0.01 ;{ }^{*} P<0.05$ vs mock control cells for each time point. 


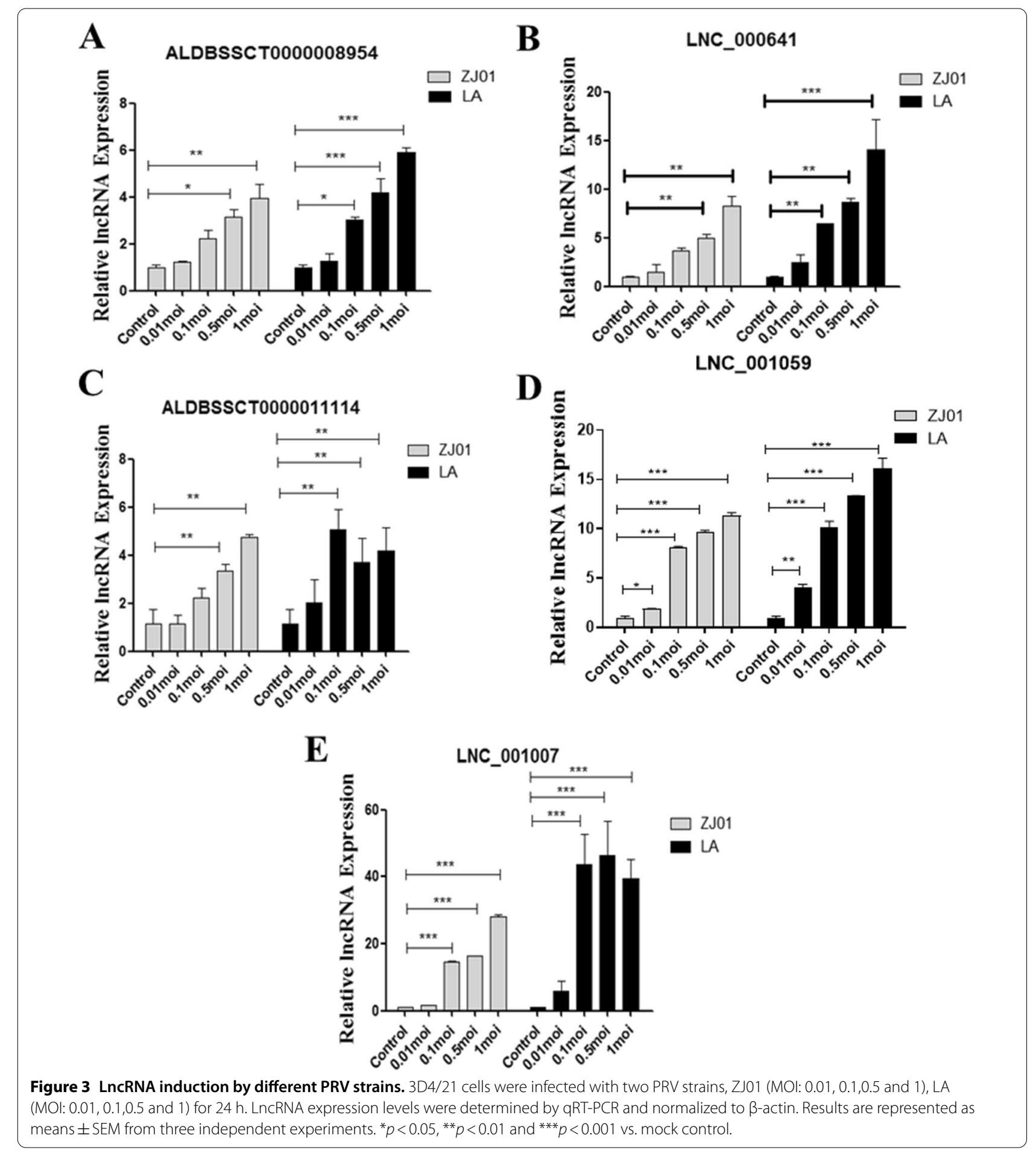

B LNC_000641

for PRV detection by $\mathrm{TCID}_{50}$, Western blot, qRTPCR and IFA. The results showed that overexpression of lnc641 significantly enhanced PRV replication (Figures 6A-E).
Lnc641 regulates PRV replication by inhibiting type I interferon

To elucidate the mechanisms of lnc641 mediated PRV replication, the location of lnc641 in cells was determined. The cytoplasmic and nuclear fractions from 

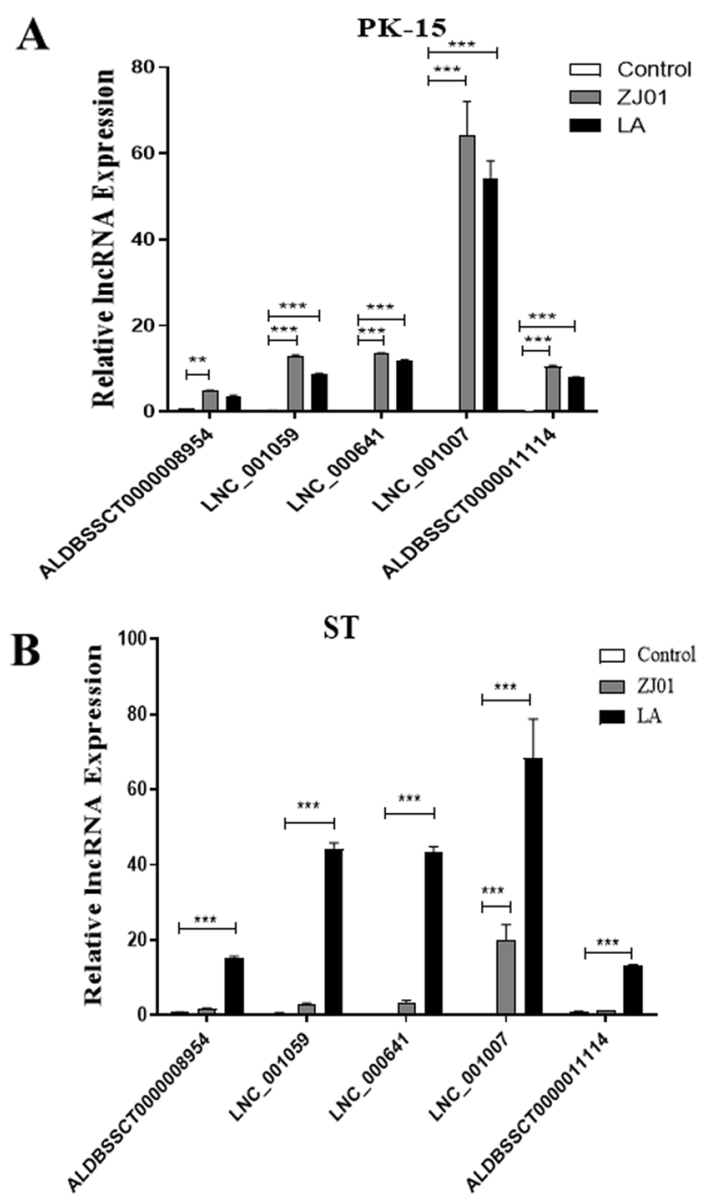

Figure 4 Lnc641 induction in PK-15 and ST cells by PRV.

PK-15 and ST cells were infected with ZJ01 and LA PRV strains at a $\mathrm{MOI}$ of 0.5 for $22 \mathrm{~h}$. LncRNA expression levels were determined by qRT-PCR and normalized to $\beta$-actin. The results are represented as means \pm SEM from three independent experiments. ${ }^{*} p<0.05$, ${ }^{* *} p<0.01$ and ${ }^{* * *} p<0.001$ vs. mock control.

3D4/21 cells were isolated and lnc641 levels in both fractions were determined by qRT-PCR. As shown in Figure $7 \mathrm{~A}, \operatorname{lnc} 641$ was enriched in the nucleus as nuclear U2snRNA, a positive control gene. Cytoplasmic $\beta$-actin mRNAs were primarily located in the cytoplasm.

To examine the role of lnc641-induced IFN expression in PRV infection, 3D4/21 cells were respectively transfected with siRNA1, siRNA2 and negative control siRNA (siNC) for $36 \mathrm{~h}$ and then infected with ZJ01 (0.01 MOI) for $24 \mathrm{~h}$, followed by qRT-PCR for IFN mRNA (Figure 7B) and Western blot for JAK/STAT protein (Figures 7C, D). The results showed that after lnc641 silencing, only IFNalpha mRNA expression increased significantly, but not IFN-beta and IFN-gamma. At the same time, knocking down of lnc641 increased the phosphorylation of JAK and STAT1 proteins.
PRV infection suppresses IFN-induced upregulation of a subset of ISGs and STAT1 phosphorylation, indicating an impairment of IFN signaling in PRV-infected cells [23]. To confirm the effect of lnc641-induced IFN expression on PRV infection, 3D4/21 cells were transfected with pcDNA3.1-641 or pcDNA3.1 $(+)$ for $24 \mathrm{~h}$ and then infected with ZJ01 (0.01 MOI) for $24 \mathrm{~h}$, followed by qRT-PCR for IFN mRNA (Figure 7E) and Western blot for JAK/STAT1 protein (Figures 7F, G). The results showed that lnc641 overexpression inhibited the IFNalpha mRNA and reduced the phosphorylated expression of JAK and STAT1.

\section{Discussion}

Pseudorabies virus is an important pathogen in the swine industry. In addition to proteins encoded by viruses, noncoding RNAs including microRNAs and lncRNAs in host cells may play an important role in viral infections [24-26]. LncRNAs represented a potential class of host factors and will be new alternatives for development of host-centered antiviral strategies. However, there were few reports on the interaction mechanism between PRV and lncRNAs [27, 28]. It was well known that lncRNAs play an important role in virus invasion and the corresponding antiviral immune response. Macrophages also played an important role in the first line of defense against pathogens invading the body. In this study, the porcine alveolar macrophage cells (3D4/21) infected with ZJ01PRV strain was used in RNA-Sequencing to identify differential lncRNAs. A total of 2424 lncRNAs were screened, of which 1320 were unannotated lncRNAs. 225 were significantly changed. Among the 126 significantly upregulated lncRNAs in ZJ01-infected cells, 5 lncRNAs were further studied. The results showed that they also induced by other another PRV strain, LA. However, the magnitude of induction of the same lncRNAs varied between ZJ01 and LA, indicating that there were differences between virus strains in induction of lncRNAs expression. Compared with the traditional strain LA, some genes of the variant strain ZJ01 were mutated. This may account for the different inducibility of lncRNA between ZJ01 and LA. But the specific genes that affect the inducibility need to be further studied. In this study, much lower MOI (0.01 and 0.5) were applied for virus infection which were entirely different from the usual doses used in different experiments. This was related to the virulence of the virus and the effect of transfection agents on cells.

LncRNAs play an important regulatory role in the battle between virus and host, involving the transcription of viral and host genes, stability and translation of mRNAs, and host antiviral response $[29,30]$. Host cells can initiate 

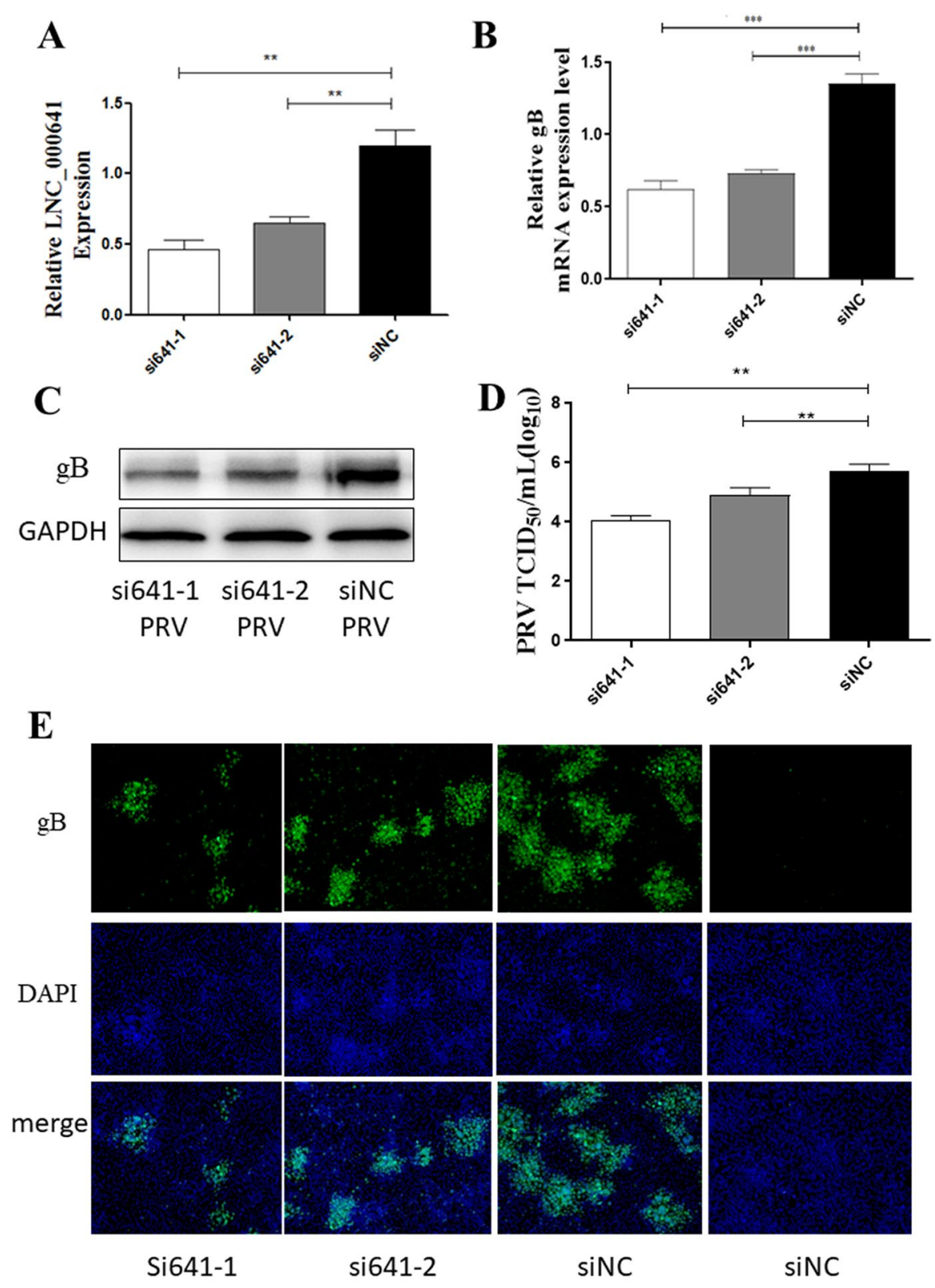

si641-2

SiNC

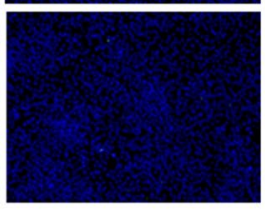

PRV

PRV

PRV

siNC

MOCK

Figure 5 Knockdown of Inc641 by siRNAs inhibits PRV replication. A 3D4/21 cells were transfected with two siRNAs (si641-1, si641-2) or negative control (siNC). At $36 \mathrm{~h}$ post-transfection, the knockdown efficiency of Inc641 was determined by qRT-PCR. B-E 3D/21 cells placed in 24-well plates were transfected with the two siRNAs for $36 \mathrm{~h}$ and then infected with ZJ01 (0.01 MOI). After $24 \mathrm{~h}$, the cell samples were collected to measure the replication of PRV by GRT-PCR $(\mathbf{B})$ and Western blot assays $(\mathbf{C})$. The supernatant was used to measure the viral titers by TCID $_{50}$ analysis (D). The cells were treated as described previously, and IFA was performed with a primary anti-gB protein monoclonal antibody to analyze the antiviral effect of PRV. Viral gB-protein is green, and nuclei are blue (E). Results are presented as means \pm SEM of data from three independent experiments. ${ }^{*} P$ value $<0.05,{ }^{* *} P<0.01,{ }^{* * *} P<0.0001$. 
A

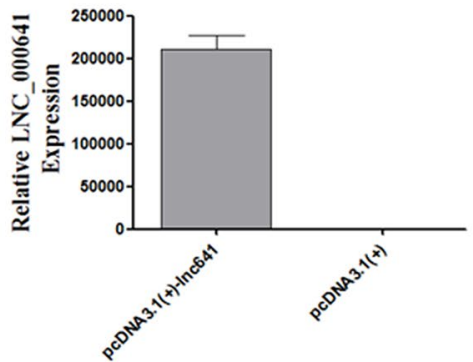

C

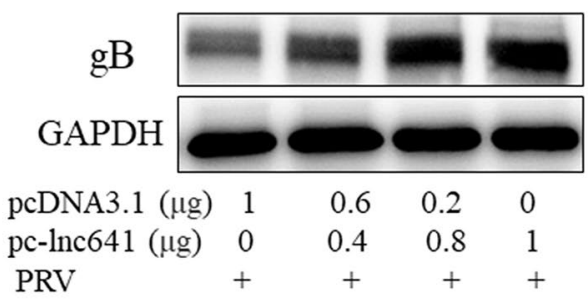

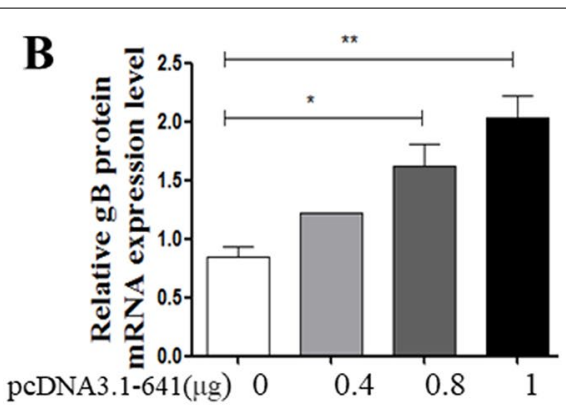

D

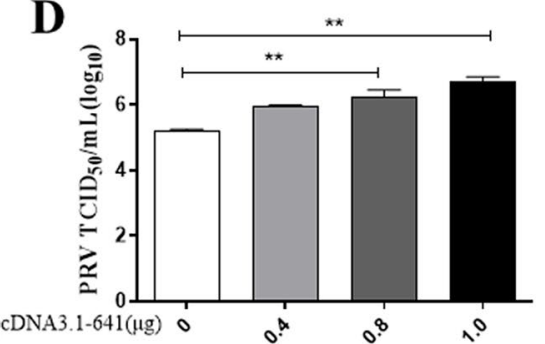

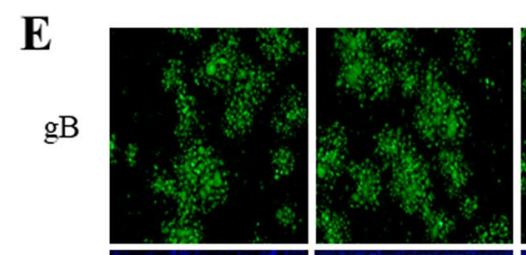
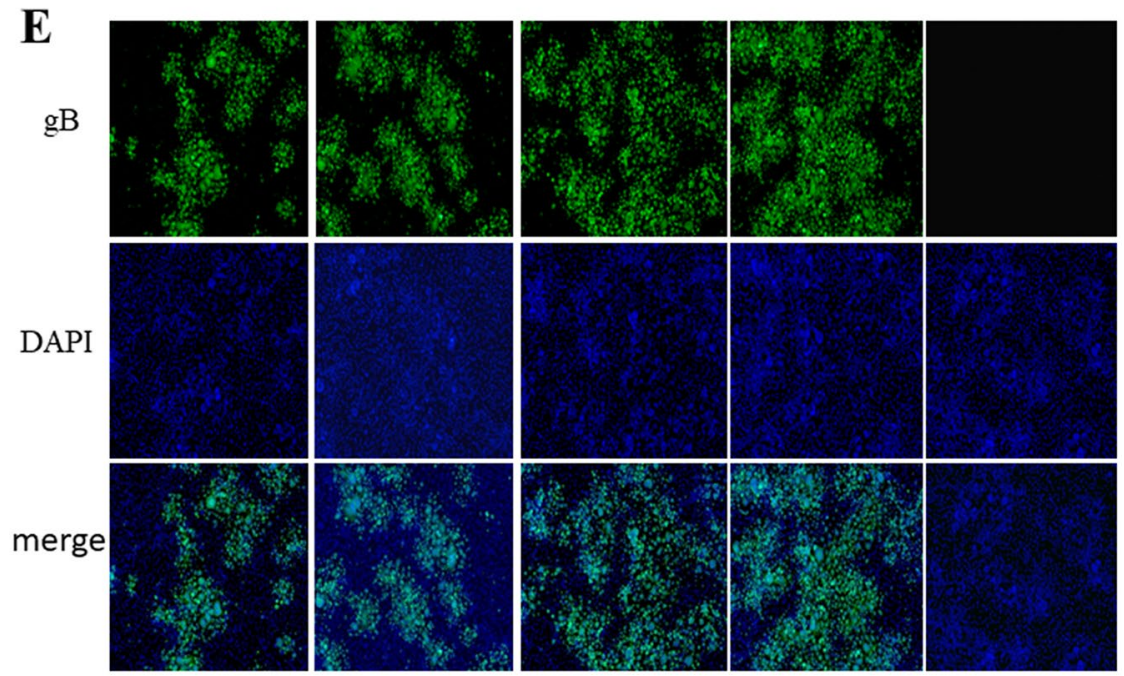

$\begin{array}{ll}\text { pcDNA3.1 }(\mu \mathrm{g}) & 1 \\ \text { pc-Inc641 }(\mu \mathrm{g}) & 0 \\ \text { PRV } & +\end{array}$
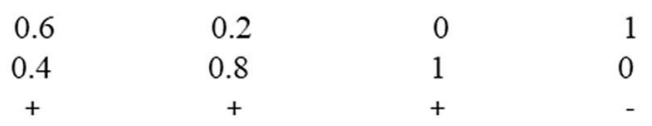

Figure 6 Lnc641 overexpression enhances PRV replication. A 3D4/21 cells were transfected with $1 \mu$ g pcDNA3.1(+)-641 or pcDNA3.1(+). At $24 \mathrm{~h}$ post-transfection, the overexpression efficiency of Inc641 was determined by qRT-PCR. B-E 3D4/21 cells were transfected with the indicated doses of pcDNA3.1(+)-641 or pcDNA3.1 (+) for $24 \mathrm{~h}$, followed by infection with ZJ01 (0.01 MOI) for $24 \mathrm{~h}$. The viral gB protein and mRNA levels were evaluated by qRT-PCR (B) and Western blot $(\mathbf{C})$. The supernatant was used to measure viral titers by TCID $_{50}$ analysis (D). The cells were treated as described previously, and IFA was performed with a primary anti-gB protein monoclonal antibody to analyze the antiviral effect of PRV. Viral gB-protein is green, and nuclei are blue (E). Results are presented as means \pm SEM of from three independent experiments. ${ }^{*} P$ value $<0.05$, ${ }^{*} P<0.01$, ${ }^{* * *} P<0.0001$. 

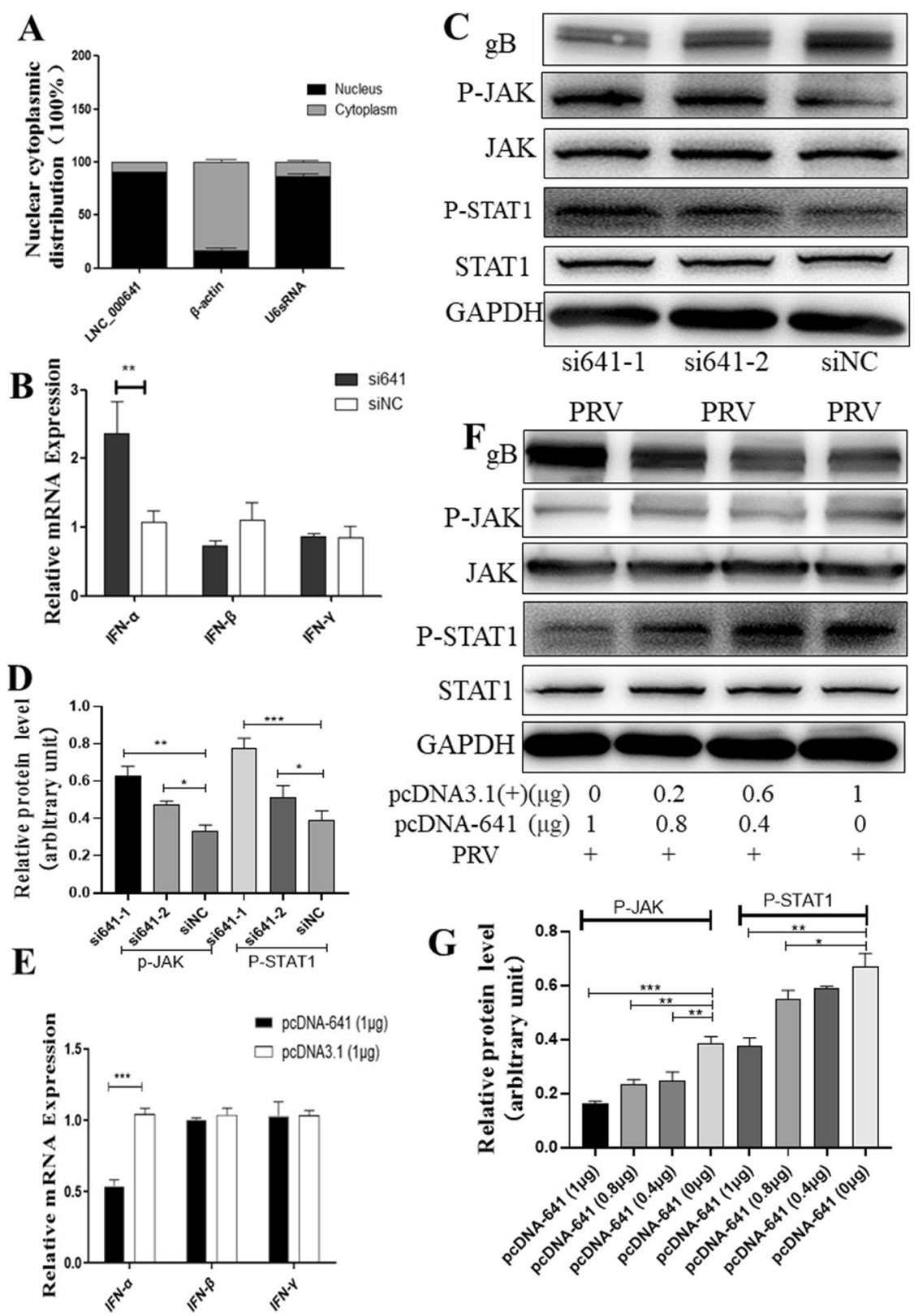

Figure 7 Lnc641 regulates PRV replication by inhibiting type I interferon. A The levels of Inc641, $\beta$-actin mRNAs (cytoplasmic RNA positive controls), and U2snRNA (nuclear RNA positive control) in cytoplasmic and nuclear fractions of 3D4/21 cells were determined by qRT-PCR. Results are represented for each gene as means \pm SEM from three independent experiments. B, C 3D4/21 cells transfected with si641 and siNC for $36 \mathrm{~h}$, then cells were infected with ZJ01 (0.01 MOI). After 24 h, IFN mRNA levels were detected by qRT-PCR. Meanwhile, the levels of JAK and STAT1 protein were detected by Western blot. D Use of Image Quant software to quantify the protein bands in Fig. 7C Western blot, as normalized to GAPDH. E 3D4/21 cells were transfected with $1 \mu \mathrm{g}$ pcDNA3.1 (+)-641 or pcDNA3.1 (+) for $24 \mathrm{~h}$, followed by infection with ZJ01 (0.01 MOI) for $24 \mathrm{~h}$. Then IFN mRNA levels were detected by qRT-PCR. F 3D4/21 cells were transfected with the indicated doses of pcDNA3.1-641 or pcDNA3.1(+) for $24 \mathrm{~h}$, followed by infection with ZJ01 (0.01 MOI) for $24 \mathrm{~h}$, the levels of JAK and STAT1 protein were detected by Western blot. G Use of Image Quant software to quantify the protein bands in $\mathbf{F}$ Western blot, as normalized to GAPDH. 
an antiviral response after viral infection by altering their own lncRNA expression. In response to virus infections, lncRNAs have been shown to modulate virus infections by diverse mechanisms [31, 32]. In this study, a lncRNA named lnc641 has been shown to promote PRV replication.

The location of lncRNA in the cell may provide important information on how to achieve its function. The lncRNA in the cytoplasm such as DANCR can compete for microRNA binding sites [33]. Another nuclear lncRNA, NRAV functions as a histone modification factor of anti-viral genes, MxA and IFITM3 [15]. The study proved that lnc641 was mainly distributed in the nucleus of 3D4/21 cells. Recently, an increasing number of lncRNAs has been reported to play roles in the innate immune response to virus infections [34, 35]. Previous studies have implicated miR-155 in host immunity against viral infections and regulation of type I IFN signaling [36, 37]. It is known that lncRNA Malat1 can inhibit the production of type I IFN in macrophages after virus infection [38], and lncRNA Sros1 can promote IFN- $\gamma-$ STAT1 mediated innate immunity [39]. Knockdown of IncRNA NONMMUT057981 can promote VSV-induced IFN production in mouse peritoneal macrophages [13]. In this study, the results demonstrated that the production of IFN-alpha and the phosphorylation of JAK and STAT1 could be regulated by knockdown and overexpression of lnc641, which indicated that lnc641 promoted the replication of PRV by regulation of IFN-alpha though JAK/STAT1 pathway. However, how lnc641 regulates the changes of JAK/STAT1 and IFN needs further research in the future.

In summary, this study determined that the lnc641 was significantly induced by PRV infection and has a profound effect on PRV replication in vitro. In addition, the results indicated that the lnc641 inhibits the innate immune response to PRV infection by down-regulating the production of IFN- $\alpha$ by inhibiting the JAK/STAT1 pathway, thereby increasing the replication of PRV. Integrated analysis showed that differentially expressed lncRNA may play a critical role in regulating PRV replication, and may provide new insights for PRV prevention and treatment strategies in the future. To illustrate the effect of lncRNAs on PRV infection, other lncRNAs will be analyzed in the following studies.

\section{Acknowledgements}

The authors thank DrYu-feng Li for sharing his extensive knowledge of transcriptomics. In addition, the critical and helpful comments from the reviewers are highly appreciated.

\section{Authors' contributions}

WX and JP conceived and designed the work. FL performed the laboratory analysis. FL, GY, and LX interpreted the data. FL, GY and BJ drafted the manuscript. WX, GY and LX revised the manuscript critically for important intellectual content. All authors read and approved the final manuscript.

\section{Funding}

This work was mainly supported by the National Key Research and Development Program of China (2016YFD0500100), grants from the Fundamental Research Funds for the Central Universities (KYZ202004), the Priority Academic Program Development of Jiangsu higher education institutions (PAPD).

\section{Availability of data and materials}

All data generated or analyzed during this study are included in this published article.

\section{Declarations}

\section{Ethics approval and consent to participate}

The experiments were approved by the Institutional Animal Care and Ethics Committee of Nanjing Agricultural University (permit no. IACECNAU20160102) and were performed in accordance with animal ethics guidelines and approved protocols.

\section{Consent to publish}

The manuscript has been approved by all authors for publication.

\section{Competing interests}

The authors declare that they have no competing interests.

\section{Author details}

${ }^{1}$ Key Laboratory of Animal Diseases Diagnostic and Immunology, Ministry of Agriculture, MOE Joint International Research Laboratory of Animal Health and Food Safety, College of Veterinary Medicine, Nanjing Agricultural University, Nanjing 210095, China. ${ }^{2}$ Jiangsu Co-Innovation Center for the Prevention and Control of Important Animal Infectious Diseases and Zoonoses, Yangzhou University, Yangzhou 225009, China.

Received: 6 December 2020 Accepted: 1 March 2021

Published online: 25 March 2021

\section{References}

1. He WT, Auclert LZ, Zhai XF, Wong G, Zhang C, Zhu HN, Xing G, Wang SL, He W, Li KM, Wang L, Han GZ, Veit M, Zhou JY, Su S (2019) Interspecies transmission, genetic diversity, and evolutionary dynamics of pseudorabies virus. J Infect Dis 219:1705-1715. https://doi.org/10.1093/infdis/ jiy 731

2. Granzow H, Weiland F, Jons A, Klupp BG, Karger A, Mettenleiter TC (1997) Ultrastructural analysis of the replication cycle of pseudorabies virus in cell culture: a reassessment. J Virol 71:2072-2082. https://doi.org/10.1128/ jvi.71.3.2072-2082.1997

3. Klupp BG, Hengartner CJ, Mettenleiter TC, Enquist LW (2004) Complete, annotated sequence of the pseudorabies virus genome. J Virol 78:424-440. https://doi.org/10.1128/jvi.78.1.424-440.2004

4. Granzow H, Klupp BG, Fuchs W, Veits J, Osterrieder N, Mettenleiter TC (2001) Egress of alphaherpesviruses: comparative ultrastructural study. J Virol 75:3675-3684. https://doi.org/10.1128/jvi.75.8.3675-3684.2001

5. Mettenleiter TC (2000) Aujeszky's disease (pseudorabies) virus: the virus and molecular pathogenesis - state of the art, June 1999. Vet Res 31:99-115. https://doi.org/10.1051/vetres:2000110

6. Brukman A, Enquist LW (2006) Suppression of the interferon-mediated innate immune response by pseudorabies virus. J Virol 80:6345-6356. https://doi.org/10.1128/jvi.00554-06

7. Iyer MK, Niknafs YS, Malik R, Singhal U, Sahu A, Hosono Y, Barrette TR, Prensner JR, Evans JR, Zhao S, Poliakov A, Cao X, Dhanasekaran SM, Wu Y-M, Robinson DR, Beer DG, Feng FY, lyer HK, Chinnaiyan AM (2015) The landscape of long noncoding RNAs in the human transcriptome. Nat Genet 47:199-208. https://doi.org/10.1038/ng.3192

8. Wang M, Yuan D, Tu L, Gao W, He Y, Hu H, Wang P, Liu N, Lindsey K, Zhang $X$ (2015) Long noncoding RNAs and their proposed functions 
in fibre development of cotton (Gossypium spp.). N Phytol 207:11811197. https://doi.org/10.1111/nph.13429

9. Wapinski O, Chang HY (2011) Long noncoding RNAs and human disease. Trends Cell Biol 21:354-361. https://doi.org/10.1016/j.tcb.2011. 04.001

10. Imam H, Bano AS, Patel P, Holla P, Jameel S (2015) The IncRNA NRON modulates HIV-1 replication in a NFAT-dependent manner and is differentially regulated by early and late viral proteins. Sci Rep 5:8639. https://doi.org/10.1038/srep08639

11. Massimelli MJ, Majerciak V, Kruhlak M, Zheng Z-M (2013) Interplay between polyadenylate-binding protein 1 and Kaposi's Sarcoma-associated herpesvirus ORF57 in accumulation of polyadenylated nuclear RNA, a viral long noncoding RNA. J Virol 87:243-256. https://doi.org/10. 1128/jvi.01693-12

12. Winterling C, Koch M, Koeppel M, Garcia-Alcalde F, Karlas A, Meyer TF (2014) Evidence for a crucial role of a host non-coding RNA in influenza A virus replication. RNA Biol 11:66-75. https://doi.org/10.4161/rna.27504

13. Jiang M, Zhang S, Yang Z, Lin H, Zhu J, Liu L, Wang W, Liu S, Liu W, Ma Y, Zhang L, Cao X (2018) Self-recognition of an inducible host IncRNA by RIG-I feedback restricts innate immune response. Cell 173:906-919.e13. https://doi.org/10.1016/j.cell.2018.03.064

14. Carnero E, Barriocanal M, Prior C, Pablo Unfried J, Segura V, Guruceaga E, Enguita M, Smerdou C, Gastaminza P, Fortes P (2016) Long noncoding RNA EGOT negatively affects the antiviral response and favors HCV replication. EMBO Rep 17:1013-1028. https://doi.org/10.15252/embr. 201541763

15. Ouyang J, Zhu X, Chen Y, Wei H, Chen Q, Chi X, Qi B, Zhang L, Zhao Y, Gao GF, Wang G, Chen J-L (2014) NRAV, a long noncoding RNA, modulates antiviral responses through suppression of interferon-stimulated gene transcription. Cell Host Microbe 16:616-626. https://doi.org/10. 1016/j.chom.2014.10.001

16. Wu J, Chen ZJ (2014) Innate immune sensing and signaling of cytosolic nucleic acids. Annu Rev Immunol 32:461-488. https://doi.org/10.1146/ annurev-immunol-032713-120156

17. Katze MG, He YP, Gale M (2002) Viruses and interferon: a fight for supremacy. Nat Rev Immunol 2:675-687. https://doi.org/10.1038/nri888

18. Decker T, Stockinger S, Karaghiosoff M, Muller M, Kovarik P (2002) IFNs and STATs in innate immunity to microorganisms. J Clin Invest 109:1271-1277. https://doi.org/10.1172/jci200215770

19. Kisseleva T, Bhattacharya S, Braunstein J, Schindler CW (2002) Signaling through the JAK/STAT pathway, recent advances and future challenges. Gene 285:1-24. https://doi.org/10.1016/s0378-1119(02)00398-0

20. Villegas VE, Zaphiropoulos PG (2015) Neighboring gene regulation by antisense long non-coding RNAs. Int J Mol Sci 16:3251-3266. https://doi. org/10.3390/ijms16023251

21. Pan Q, Zhao Z, Liao Y, Chiu S-H, Wang S, Chen B, Chen N, Chen Y, Chen J-L (2019) Identification of an interferon-stimulated long noncoding RNA (LncRNA ISR) involved in regulation of influenza A virus replication. Int J Mol Sci 20:5118. https://doi.org/10.3390/ijms20205118

22. More S, Zhu Z, Lin K, Huang C, Pushparaj S, Liang Y, Sathiaseelan R, Yang X, Liu L (2019) Long non-coding RNA PSMB8-AS1 regulates influenza virus replication. RNA Biol 16:340-353. https://doi.org/10.1080/15476286. 2019.1572448

23. Jin L, Scherba G (1999) Expression of the pseudorabies virus latencyassociated transcript gene during productive infection of cultured cells. J Virol 73:9781-9788. https://doi.org/10.1128/jvi.73.12.9781-9788.1999

24. Li Y, Zheng G, Zhang Y, Yang X, Liu H, Chang H, Wang X, Zhao J, Wang C, Chen L (2017) MicroRNA analysis in mouse neuro-2a cells after pseudorabies virus infection. J Neurovirol 23:430-440. https://doi.org/10.1007/ s13365-016-0511-y
25. Liu F, Zheng H, Tong W, Li G-X, Tian Q, Liang C, Li L-W, Zheng X-C, Tong G-Z (2016) Identification and analysis of novel viral and host dysregulated MicroRNAs in variant pseudorabies virus-infected PK15 cells. PLoS One 11:e0151546. https://doi.org/10.1371/journal.pone.0151546

26. Landeras-Bueno S, Ortin J (2016) Regulation of influenza virus infection by long non-coding RNAs. Virus Res 212:78-84. https://doi.org/10.1016/j. virusres.2015.08.008

27. Jin Y, Zhang K, Huang W, Tang W, Li H, Dong W, Gu J, Zhou J (2020) Identification of functional IncRNAs in pseudorabies virus type II infected cells. Vet Microbiol 242:108564. https://doi.org/10.1016/j.vetmic.2019.108564

28. Guan X, Liu J, Jiang H, Wu C-X, Chen H-C, Liu Z-F (2018) Expression of pseudorabies virus-encoded long noncoding RNAs in epithelial cells and neurons. J Neurovirol 24:597-605. https://doi.org/10.1007/ s13365-018-0651-3

29. Liu W, Ding C (2017) Roles of LncRNAs in viral infections. Front Cell Infect Microbiol 7:205. https://doi.org/10.3389/fcimb.2017.00205

30. Fortes $P$, Morris KV (2016) Long noncoding RNAs in viral infections. Virus Res 212:1-11. https://doi.org/10.1016/j.virusres.2015.10.002

31. Wang Z, Fan P, Zhao Y, Zhang S, Lu J, Xie W, Jiang Y, Lei F, Xu N, Zhang Y (2017) NEAT1 modulates herpes simplex virus-1 replication by regulating viral gene transcription. Cell Mol Life Sci 74:1117-1131. https://doi.org/10 1007/s00018-016-2398-4

32. Gomez JA, Wapinski OL, Yang YW, Bureau J-F, Gopinath S, Monack DM, Chang HY, Brahic M, Kirkegaard K (2013) The NeST long ncRNA controls microbial susceptibility and epigenetic activation of the interferongamma locus. Cell 152:743-754. https://doi.org/10.1016/j.cell.2013.01.015

33. Yuan S-X, Wang J, Yang F, Tao Q-F, Zhang J, Wang L-L, Yang Y, Liu H, Wang Z-G, Xu Q-G, Fan J, Liu L, Sun S-H, Zhou W-P (2016) Long noncoding RNA DANCR increases stemness features of hepatocellular carcinoma by derepression of CTNNB1. Hepatology 63:499-511. https://doi.org/10. 1002/hep.27893

34. Ma Y, Ouyang J, Wei J, Maarouf M, Chen J-L (2017) Involvement of host non-coding RNAs in the pathogenesis of the influenza virus. Int J Mol Sci 18:39. https://doi.org/10.3390/ijms18010039

35. Ouyang J, Hu J, Chen J-L (2016) IncRNAs regulate the innate immune response to viral infection. Wiley Interdiscip Rev RNA 7:129-143. https:/ doi.org/10.1002/wrna.1321

36. Harrison EB, Emanuel K, Lamberty BG, Morsey BM, Li M, Kelso ML, Yelamanchili SV, Fox HS (2017) Induction of miR-155 after brain injury promotes type 1 interferon and has a neuroprotective effect. Front Mol Neurosci 10:228. https://doi.org/10.3389/fnmol.2017.00228

37. Wang B, Fu M, Liu Y, Wang Y, Li X, Cao H, Zheng SJ (2018) gga-miR-155 enhances type I interferon expression and suppresses infectious burse disease virus replication via targeting SOCS1 and TANK. Front Cell Infect Microbiol 8:55. https://doi.org/10.3389/fcimb.2018.00055

38. Liu W, Wang Z, Liu L, Yang Z, Liu S, Ma Z, Liu Y, Ma Y, Zhang L, Zhang X, Jiang M, Cao X (2020) LncRNA Malat1 inhibition of TDP43 cleavage suppresses IRF3-initiated antiviral innate immunity. Proc Natl Acad Sci U S A 117:23695-23706. https://doi.org/10.1073/pnas.2003932117

39. Xu H, Jiang Y, Xu X, Su X, Liu Y, Ma Y, Zhao Y, Shen Z, Huang B, Cao X (2019) Inducible degradation of IncRNA Sros 1 promotes IFN-gamma-mediated activation of innate immune responses by stabilizing Stat1 mRNA. Nat Immunol 20:1621-1630. https://doi.org/10.1038/s41590-019-0542-7

\section{Publisher's Note}

Springer Nature remains neutral with regard to jurisdictional claims in published maps and institutional affiliations. 